\title{
Cytomegalovirus Retinitis in a Patient on Long-term Mycophenolate Mofetil Treatment for Myasthenia Gravis
}

\author{
Shyam Patel, MD, Alexander Robin, BS \\ Department of Ophthalmology, Cook County Hospital, Chicago, IL, USA \\ ORCID: \\ Shyam Patel: 0000-0002-4858-3423
}

J Ophthalmic Vis Res 2020; 15 (3): 428-431

\section{PRESENTATION}

A 64-year-old man with myasthenia gravis (MG) presented with blurry vision in his left eye (OS). His visual acuity was $20 / 20$ in the right eye and $20 / 50$ OS. His intraocular pressures, pupils, and anterior segment were normal. He had 1+ vitritis and vaso-occlusive appearance of the retina with sclerotic vessels and hemorrhages in the inferonasal quadrant OS (Figure 1), consistent with features of cytomegalovirus (CMV) retinitis. He was immunocompromised secondary to mycophenolate mofetil (MMF) administered for $\mathrm{MG}$, with $0.6 \%$ lymphocytes (normal: 26.0-46.0\%), a lymphocyte count of $0.1 \times 10^{3}$ cells $/ \mu \mathrm{L}$, and a leukocyte count of $11.3 \times 10^{3}$ cells/ $\mu \mathrm{L}$. He was receiving $1000 \mathrm{mg}$ of MMF BID. The human immunodeficiency virus (HIV) test result was negative, and no further workup for immunosuppression, including cancer, was conducted.

The patient was administered with $0.05 \mathrm{~mL}$ ganciclovir (4 mg/0.1 mL) and $0.10 \mathrm{~mL}$ foscarnet $(2.4 \mathrm{mg} / 0.1 \mathrm{~mL})$ intravitreal injections on diagnosis. Subsequently, his symptoms improved, and

Correspondence to:

Shyam Patel, MD. Department of Ophthalmology, Cook County Hospital, 1900 West Polk St., Ste. 617, Chicago, 60612, IL, USA.

Email: spatel0687@gmail.com

Received: 01-04-2018Ａccepted: 19-06-2019

Access this article online

Website: https://knepublishing.com/index.php/JOVR

DOI: 10.18502/jovr.v15i3.7463 a 10-week course of oral valganciclovir (900 $\mathrm{mg}$ BID for 21 days followed by $900 \mathrm{mg}$ QD for seven weeks) was administered. There was also a decrease in the dosage and eventual cessation of MMF with initiation of intravenous immunoglobulins. His lymphocytes improved to $9.8 \%$ (lymphocyte count, $0.7 \times 10^{3}$ cells $/ \mu \mathrm{L}$; leukocyte count, $6.9 \times 10^{3}$ cells $\left./ \mu \mathrm{L}\right)$.

On valganciclovir discontinuation in week 10 , the patient had a visual acuity of 20/25 OS with no inflammation and improvements in retinal hemorrhages and lesions. An epiretinal membrane was observed on macular optical coherence tomography (Figure 2). The inferonasal retina showed inactive whitish atrophy (Figure 3).

\section{DISCUSSION}

CMV retinitis is the most common ocular opportunistic infection associated with acquired immune deficiency syndrome. ${ }^{[1]}$ The prevalence of CMV retinitis in HIV patients has decreased since the advent of highly active antiretroviral therapy (HAART). ${ }^{[2]}$ However, the rate of CMV retinitis in non-HIV patients is increasing, likely due to the use of aggressive immunosuppressive agents. ${ }^{[1]} \mathrm{CMV}$ is an infectious complication frequently associated with MMF. ${ }^{[3]}$

This is an open access journal, and articles are distributed under the terms of the Creative Commons Attribution-NonCommercial-ShareAlike 4.0 License, which allows others to remix, tweak, and build upon the work non-commercially, as long as appropriate credit is given and the new creations are licensed under the identical terms.

How to cite this article: Patel S, Robin A. Cytomegalovirus Retinitis in a Patient on Long-term Mycophenolate Mofetil Treatment for Myasthenia Gravis. J Ophthalmic Vis Res 2020;15:428-431. 


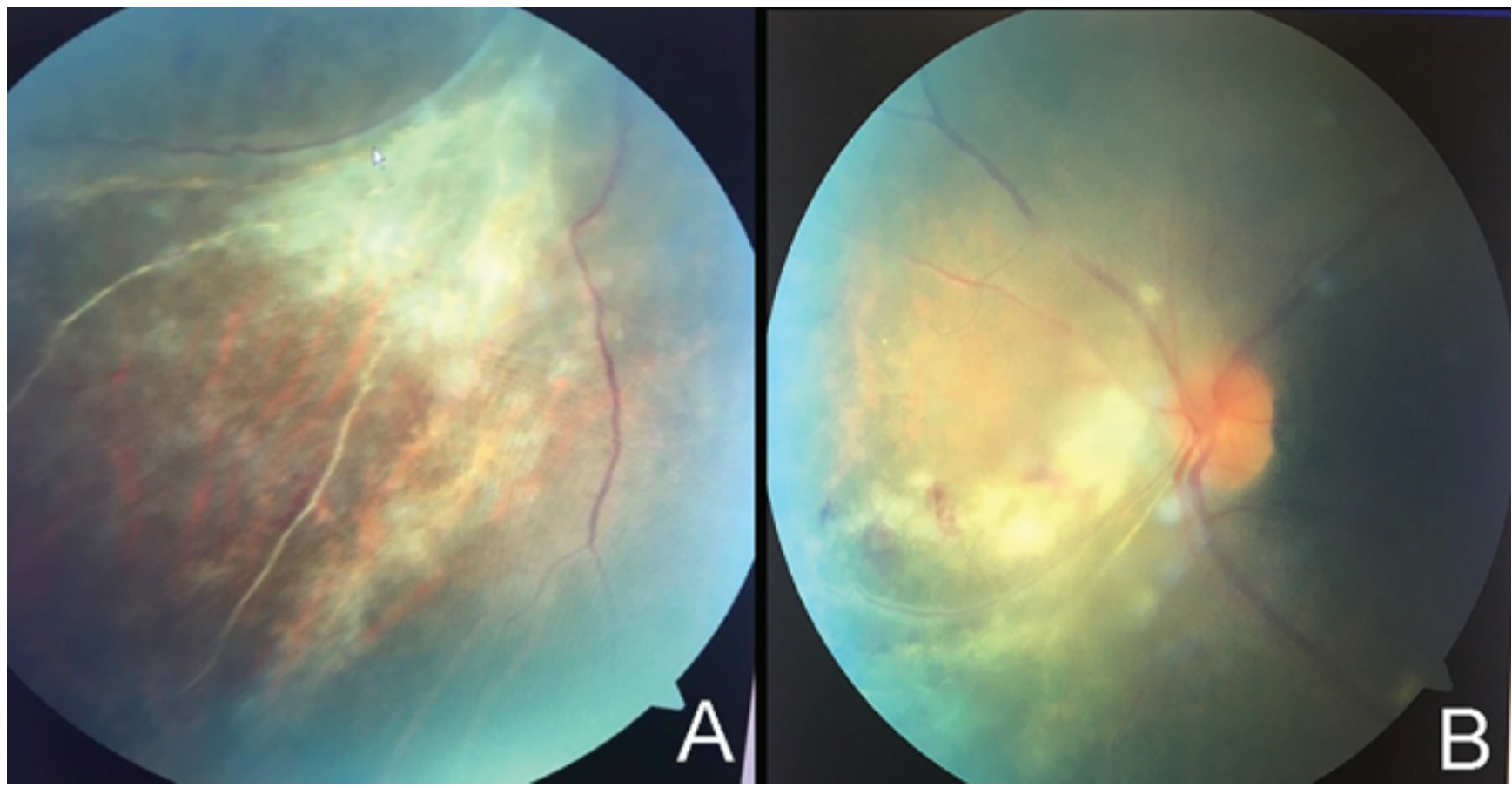

Figure 1. (A) A funduscopic photo of the left eye shows fluffy white lesions with intraretinal hemorrhage predominantly in the inferonasal quadrant. (B) A funduscopic photo of the left inferonasal quadrant five weeks after treatment initiation.

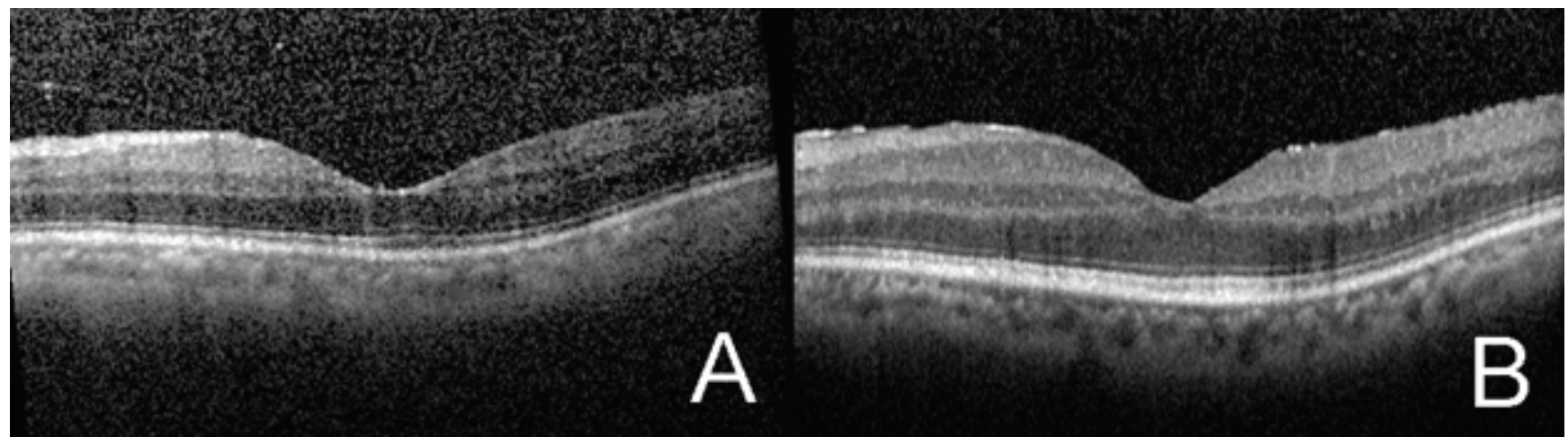

Figure 2. (A) OCT of the macula of the left eye before treatment initiation showing vitreomacular adhesion and few vitreous cells. (B) OCT of the macula of the left eye after 10 weeks from treatment initiation showing a fine epiretinal membrane.

Visual prognosis of CMV infection in nonHIV patients is similar to that in HIV patients with poor visual outcomes associated with retinal detachments and macular involvement. ${ }^{[1]}$ CMV retinitis in patients with concomitant HIV infection lacks vitreous involvement. ${ }^{[2]}$ In contrast, vitritis is more commonly associated with non-HIV-related CMV retinitis infections. ${ }^{[2]}$ This is consistent with our patient's presentation.

CMV retinitis treatment in HIV patients involves HAART and antiviral therapy. ${ }^{[1]}$ In non-HIV patients, different etiologies of an immunocompromised state must be considered. Commonly used treatment strategies include systemic ganciclovir, foscarnet, valganciclovir, and intravitreal ganciclovir. Our patient received one initial intravitreal injection each of ganciclovir and foscarnet, as well as oral valganciclovir. Intravitreal injections are important for the treatment of vision-threatening CMV infections and were used in our case. ${ }^{[4]}$ Nevertheless, the mainstay treatment of CMV retinitis remains systemic antivirals, and it is not always necessary to start with intravitreal injections. The combination of intravitreal ganciclovir 


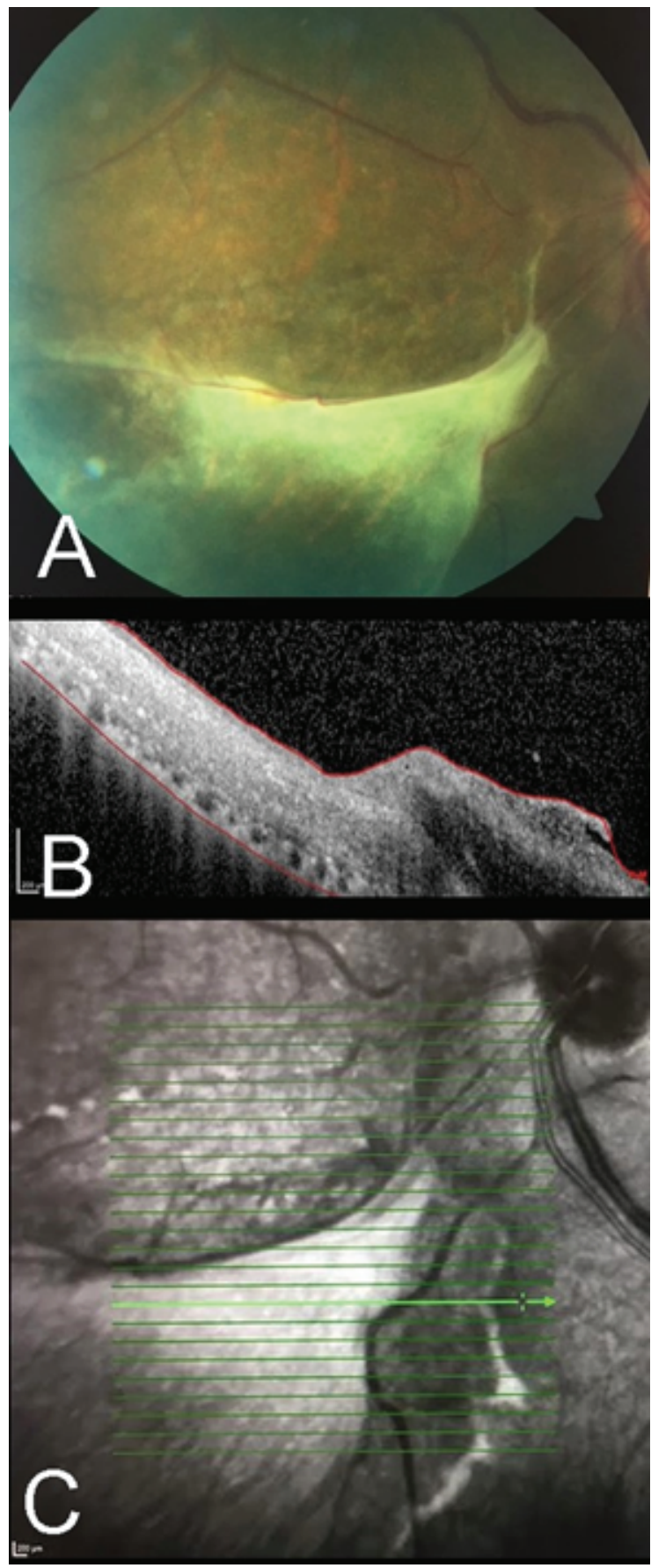

Figure 3. The inferonasal fundus photo of the left eye at 10 weeks showing preretinal fibrosis with a corresponding OCT (over the white lesion, see Arrow) showing an atrophic retina with resolved vitritis. A corresponding infrared image showing the line of scan of the OCT.

and foscarnet is effective in treating CMV retinitis. ${ }^{[5]}$

In summary, we presented a patient with MG who developed CMV retinitis due to immunosuppression as a result of MMF treatment. He was treated successfully with intravitreal and systemic antivirals.

\section{Financial Support and Sponsorship}

Nil.

\section{Conflicts of Interest}

There are no conflicts of interest. 


\section{REFERENCES}

1. Kim D, Jo J, Joe S, Kim J, Yoon Y, Lee J. Comparison of visual prognosis and clinical features of cytomegalovirus retinitis in HIV and non-HIV patients. Retina 2017;37:376381.

2. Iu L, Fan M, Lau J, Chan T, Kwong Y, Wong I. Longterm follow-up of Cytomegalovirus Retinitis in non-HIV immunocompromised patients: clinical features and visual prognosis. Am J Ophthalmol 2016;165:145-153.
3. Meier-Kriesche HU, Friedman G, Jacobs M, Mulgaonkar S, Vaghela M, Kaplan B. Infectious complications in geriatric renal transplant patients: comparison of two immunosuppressive protocols. Transplantation 1999;68:1496-1502.

4. Pearce W, Yeh S, Fine H. Management of Cytomegalovirus Retinitis in HIV and non-HIV patients. Ophthalmic Surg Lasers Imaging Retina 2016;47:103-107.

5. Velez G, Roy CE, Whitcup SM, Robinson MR. High-dose intravitreal ganciclovir and foscarnet for cytomegalovirus retinitis. Am J Ophthalmol 2001;131:396-397. 